\title{
Use of Intelligent Voice Assistants by Older Adults with Low Technology Use
}

\author{
ALISHA PRADHAN and AMANDA LAZAR, University of Maryland \\ LEAH FINDLATER, University of Washington
}

\begin{abstract}
Voice assistants embodied in smart speakers (e.g., Amazon Echo, Google Home) enable voice-based interaction that does not necessarily rely on expertise with mobile or desktop computing. Hence, these voice assistants offer new opportunities to different populations, including individuals who are not interested or able to use traditional computing devices such as computers and smartphones. To understand how older adults who use technology infrequently perceive and use these voice assistants, we conducted a 3-week field deployment of the Amazon Echo Dot in the homes of seven older adults. While some types of usage dropped over the 3-week period (e.g., playing music), we observed consistent usage for finding online information. Given that much of this information was health-related, this finding emphasizes the need to revisit concerns about credibility of information with this new interaction medium. Although features to support memory (e.g., setting timers, reminders) were initially perceived as useful, the actual usage was unexpectedly low due to reliability concerns. We discuss how these findings apply to other user groups along with design implications and recommendations for future work on voice-user interfaces.
\end{abstract}

CCS Concepts: • Human-centered computing $\rightarrow$ Human computer interaction (HCI); Empirical studies in HCI;

Additional Key Words and Phrases: Conversational interfaces, voice assistants, smart speakers, low technology use, older adults

\section{ACM Reference format:}

Alisha Pradhan, Amanda Lazar, and Leah Findlater. 2020. Use of Intelligent Voice Assistants by Older Adults with Low Technology Use. ACM Trans. Comput.-Hum. Interact. 27, 4, Article 31 (September 2020), 27 pages.

https://doi.org/10.1145/3373759

\section{INTRODUCTION}

Intelligent voice assistants embodied in smart speakers such as the Amazon Echo and Google Home have brought conversational, ${ }^{1}$ voice-based interaction into the mainstream. Because these

\footnotetext{
${ }^{1}$ We do acknowledge that current day voice assistants are not entirely "conversational"-as often indicated in prior works [59], yet in this article we refer them as "conversational" to differentiate this form of voice-based interaction that makes

This work was partially supported by the Mozilla Foundation and the National Science Foundation under award \#1816145. Authors' addresses: A. Pradhan and A. Lazar, College of Information Studies, University of Maryland, 4130 Campus Dr, College Park, MD 20740, United States; emails: \{alishapr, lazar\}@umd.edu; L. Findlater, Human Centered Design and Engineering, University of Washington, 3960 Benton Lane NE, Seattle, WA 98195, United States; email: leahkf@uw.edu.

Permission to make digital or hard copies of all or part of this work for personal or classroom use is granted without fee provided that copies are not made or distributed for profit or commercial advantage and that copies bear this notice and the full citation on the first page. Copyrights for components of this work owned by others than ACM must be honored. Abstracting with credit is permitted. To copy otherwise, or republish, to post on servers or to redistribute to lists, requires prior specific permission and/or a fee. Request permissions from permissions@acm.org. (C) 2020 Association for Computing Machinery.

1073-0516/2020/09-ART31 \$15.00

https://doi.org/10.1145/3373759
}

ACM Transactions on Computer-Human Interaction, Vol. 27, No. 4, Article 31. Publication date: September 2020. 
voice assistants offer non-visual interaction, they present new possibilities for accessibility compared to traditional computers and touchscreen mobile devices. Emphasizing this potential, researchers have begun investigating the use of smart speakers by people with physical, sensory, and cognitive disabilities, finding that voice interaction is seen as particularly valuable by blind users and individuals with mobility impairments [61]. Conversational voice assistants embodied in these smart speakers can also be seen as approachable, with users viewing them as "more natural to interact with" than other computing devices [61].

In parallel with these advances, HCI researchers have become increasingly interested in understanding how technologies might be more useful for older people. Past research has been critiqued for characterizing older adults as uniformly uninterested in or unable to use technology [82]. Older adults are a highly diverse group, with individual experiences affected by gender, class, ethnicity, and other factors. While older adults as a whole are the fastest growing group of technology users, internet use varies greatly by age, income, and education [3]. In the United States, for example, less than half of older adults who have at most a high school diploma report using the internet [3]. For older adults who do not use technology regularly, the accessibility and approachability of conversational voice-based interfaces may offer new opportunities.

In this article, we aim to answer the following questions: (1) How do older adults who do not regularly use a computing device perceive intelligent voice assistants embodied in smart speakers? (2) What do they use these devices for? (3) What challenges arise from the use of these systems and how can those challenges be addressed? To answer these questions, we conducted a study by deploying Amazon Echo Dot devices-a smart speaker with the Alexa voice assistant-in seven households with older adults who used digital technology infrequently. We studied participants' usage over a period of 3 weeks using a range of data sources, including usage logs from the paired Amazon Alexa app, self-reported data from semi-structured interviews, and daily diary entries.

We found that all participants used the voice-based smart speaker to seek a variety of online information, such as questions related to health, local businesses, and food and drink. More than half of the participants reported that the ease of use led them to seek more information online using these devices. Device features for supporting memory (e.g., reminders, timers) were less frequently used than initial positive comments about these features had led us to expect. Concerns about reliability of these features arose due to reasons such as forgetting to set reminders on the device and technological dependencies on unstable infrastructure (e.g., electricity, internet connectivity). Most participants in our study found the voice-interface easier to use than traditional computing devices, suggesting that voice-based interfaces can enable easy access to digital technology. Overall, even though voice-based interaction appeared to be easy to learn, the following issues exist: devices timed out before completion of voice commands, and there are unclear and inconsistent voice commands that must be remembered. Other challenges include dependency on paired computing devices and awareness of device capabilities.

This article makes the following contributions: (1) the first in-depth deployment study on initial usage patterns of an embodied voice assistant by older adults who use technology infrequently; (2) identification of benefits (e.g., approachability) and challenges (e.g., how to convey credibility of online information) for voice-based information access for this user group; (3) recommendations for design and future work on making voice-based interfaces more accessible and useful for this population.

use of more natural and intuitive spoken language as input than early research prototypes of voice-based interaction that have often used stringent keywords [60,79] 


\section{RELATED WORK}

Our study is informed by research on traditional digital technology adoption by older adults, design and use of voice-based interfaces by older adults, as well as on the growing body of work on voice assistants embodied in smart speakers in the home.

\subsection{Technology Adoption and Older Adults}

Often studies examining technology use by older adults consider them to be a homogeneous population, positioning them negatively [82]. Although a number of counterexamples have emerged in the recent years, such as analyses of older hackers [77], bloggers [13, 38], and individuals eager to engage with new technologies and envision design futures [65], much of the literature still associates older adults with being slower, more anxious, and less competent with technology than younger populations [82], less likely to use computers [17], smartphones [54], and the internet [5] than younger adults. Yet, older adults represent the fastest growing group of digital computing technologies [3]. In this article, aligned with a perspective that recognizes the diversity in the older adult population in terms of technology use, we focus on a particular segment, i.e., individuals who might experience barriers or disinterest in using digital technology. Thus, we explicitly recruit older adults who do not use computing devices frequently (representative of about onefourth of older internet users in the United States in 2017 [4]) to ensure that their perspectives can inform the design of voice-based personal assistant technologies. Below, we discuss barriers to technology adoption for older adults identified in previous literature.

Some older people can encounter barriers to technology use due to factors such as lack of digital technology experience, age related factors, and poorly designed technologies. Previous experience with using digital technology (e.g., in the workplace before retirement) and internet-related knowledge can impact technology usage by older adults [16, 40,72], for example, correlating positively with performance on internet-based information retrieval tasks [16]. Usability and other issues such as lack of intuitive interfaces also pose barriers to technology use. Aula [6] identified navigation issues due to the website structure (e.g., getting back to previous page) and difficulties in understanding the terminology (e.g., warning messages) can be problematic for older adults using the internet for web-based search tasks. The lack of intuitiveness of visual interaction elements (e.g., icons, scrollbar) can also make it difficult for older adults to use traditional digital technology [26]. Perhaps resulting in part from these user interface barriers, older adults have perceived digital technology as complex to learn and use [23, 80], possibly creating attitudinal barriers such as lower self-efficacy and anxiety of using computers [17, 40], distrust toward adopting technology [37], as well as frustration [25]. The perceived intrusiveness of the current day digital technology also discourages some older people from adopting new technologies [42]. Finally, the large initial cost of computing devices is also a factor impeding adoption among older people [40, 80].

Accessibility issues also contribute to technology adoption resistance. Technology can be inaccessible for people who experience changes that commonly occur with aging. Piper et al. [56] found that late-life vision loss presents challenges to technology use due to difficulties learning new accessibility tools such as screen readers. Moreover, performance on internet-based tasks depends on cognitive abilities, such as reasoning, working memory, and perceptual speed [72]. When technologies are not designed taking into account typical age-related changes in perception and processing, they may become inaccessible or difficult to use for a substantial segment of the population. Yet, much of these prior works related to older adults' technology resistance has focused on graphical user interfaces (GUIs), comprising of traditional computing devices such as desktop or laptop computers, smartphones or tablets. As new technologies such as voiceuser interfaces (VUIs) become widespread, there is a need to understand how these technologies 
cater to the unique needs and desires of older adults who do not regularly use traditional digital technologies.

\subsection{Voice-Based Interfaces for Older Adults}

Voice-based interaction has been employed to support older adults in a variety of contexts. Much of this work has explored using voice interaction for controlling smart homes $[60,79]$, assistive and social robots $[7,14,66]$, and cognitive and other multimodal assistants [21, 58, 88]. Multimodal interactions including voice have also been studied for older adults using traditional computing devices such as computers [67] and mobile phones [30, 75]. Sato et al. [67] developed a "voicebased augmented interface" to support use of web applications, by reading aloud confirmations of the user's input (e.g., text entry), notification of status change on a webpage (e.g., page loading), and suggesting possible actions to perform, along with explaining choices available on a page. Older adults using this augmented interface for banking and online shopping tasks mentioned an increase in overall confidence of using web-based applications, reflecting the potential of voicebased interfaces for encouraging older adults in using web-based services. Further, findings from Schlogl et al.'s [70] lab-based comparative study on using VUIs such as Apple's Siri and GUIs for writing emails suggest that older adults actually preferred Siri (a smartphone-based voice assistant) to the standard GUI. Similarly in another study, voice interaction was paired with visual output on mobile devices and older adults using the prototype perceived it to be easy to use and useful because of the hands-free nature of the interaction [30]. At the same time, challenges can arise with voice interaction due to hearing loss, noisy environments, and concerns around privacy [30].

This body of work suggests that VUIs have the potential for lowering the technology adoption barriers that are present with more traditional digital technologies. However, much of the research on VUIs for older adults has studied voice interaction to augment an existing visual display, rather than focusing only on the voice modality. Recently, researchers have started exploring voice-only interfaces without a visual display for older adults in the context of email tasks [11] and a voicebased online community [12]. These works primarily focus on connecting older adults but have not examined providing access to digital information.

In a study conducted by Wulf et al. [89], older adults (aged 65+) used a smartphone-based voice assistant (iOS's Siri) to perform basic tasks in a single-session study, such as checking the weather or asking directions. Participants appreciated the speed of voice interaction and lack of typing, although issues arose due to speech recognition and internet connectivity. These participants were almost all daily smartphone/tablet and computer users. Similar to Wulf et al., our work also examines the use of a voice-based assistant embodied in smart speakers (in contrast to smartphones) by older adults. However, unlike this work and the existing body of work on older adults and voice-user interfaces that have solely identified the potential of VUIs toward enabling easier digital technology access, we aim to extend the existing body of knowledge by investigating the use of voice-only interfaces by digitally inactive older adults (i.e., who do not use computing technology regularly), older adults who constitute one-quarter of total older internet users (in United States, 2017) [4]. In addition, we add to an emerging understanding of how perceptions change over time through a 3-week field deployment of a voice-only interface in users' home environments.

\subsection{Voice Assistants in the Home}

Most work on smart speaker-based voice assistants (e.g., Amazon's Alexa, Google Assistant) in the home environment has focused on how the general population uses these devices [2], such as understanding privacy and security concerns due to the "always on," voice-activated nature of the devices [1, 24, 52, 90]. Researchers have also looked at social aspects of these voice-based intelligent agents, studying anthropomorphism of the embodied voice assistants, finding, for example, 
that users in multi-user households where social interaction occurs around the smart speaker are more likely to personify the agent than users living in single-user households [62]. Other findings indicate that the personification of Alexa, indicated by instances such as users thanking the device or exhibiting politeness in speech (e.g., "please"), is not explicitly intended by the user, but is rather a "social mindless response" [43]. Researchers have also deployed smart speaker devices in multi-user households to understand how these technologies fit into the complex social home environment $[8,59]$; use of the device is often concurrent with other activities, such as conversations during dinner, and issues around the power dynamics of device control can arise [59]. Different applications of smart speakers are also being explored, such as an exercise reminder [76], for inclusive education of children with mixed abilities [48], and as a publicly available internet connection for people in low-resource environments of developing countries [64], overall indicating an increasing popularity of this new technology.

Although much of the work on smart speakers has focused on its use by general populations, some researchers have also examined smart speaker use by specific user groups, including children [19], English language learners [18], and people with disabilities [61]. In an analysis of reviews of the Amazon Echo that mentioned use by people with disabilities, Pradhan et al. [61] found that $13.3 \%$ of the reviews in their dataset included older adults as users of this technology, and many of these identified that the device provided easy access to digital technology. This preliminary finding suggests that this technology may be useful for older adults; however, older adults were not the focus of the study, and most of these reviews were written by a family member rather than by older adults themselves. Further emerging work in conversational user interfaces is calling attention to the need to investigate voice assistants' response design with respect to the user's request, and anthropomorphic design particularly for aging populations [68]. Our study contributes to this growing interest in understanding older adults' use of voice assistants and expands on this prior work by investigating the perceptions and use of smart speakers by digitally inactive older adults who are infrequent technology users.

\section{METHODS}

To understand how older adults who use technology infrequently perceive and use voice-based conversational interaction, we conducted a 3-week field deployment of a smart speaker (Amazon Echo Dot) with seven individuals. Our method triangulates data through weekly semi-structured interviews, daily diary entries, and usage logs.

\subsection{Participants}

Seven ${ }^{2}$ participants (one male, seven female) aged 65 or older who had no prior experience of using voice assistants, and who used a digital computing device such as a computer, smartphone or tablet less than once a day took part in this study. To characterize low usage of digital computing devices, we chose a usage frequency of less than once a day since most people who use the internet go online at least once a day (about $88 \%$ of all internet users) [53]. All participants had their own wireless internet connection (a requirement to participate) because it came bundled with their cable TV connection. Recruitment was done through local independent living organizations, snowball sampling, and word of mouth. At the end of the study, participants kept the study devices (Amazon Echo Dot and a Fire tablet) as compensation.

Five participants lived at independent living facilities, three of whom (P1-P3) lived in a lowincome facility (household earning less than $50 \%$ of area median income). Two participants lived

\footnotetext{
${ }^{2}$ Eight participants originally consented to participate in the study. One participant dropped out after the first week due to difficulty finding time for weekly interviews.
} 
Table 1. Participant Demographic and Computing Use

\begin{tabular}{|l|c|l|l|l|l|}
\hline ID & Age & Gender & \multicolumn{1}{|c|}{ Education } & \multicolumn{1}{|c|}{$\begin{array}{l}\text { Computing Devices and } \\
\text { Internet Usage }\end{array}$} & \multicolumn{1}{c|}{$\begin{array}{l}\text { Computing } \\
\text { Confidence }\end{array}$} \\
\hline P1 & 65 & Male & High school & $\begin{array}{l}\text { Computer, every few days to play chess or check } \\
\text { social media; computer is primarily used by } \\
\text { grandchildren. }\end{array}$ & $\begin{array}{l}\text { Not at all } \\
\text { confident }\end{array}$ \\
\hline P2 & 75 & Female & High school & $\begin{array}{l}\text { Computer, once a week to play games or check } \\
\text { email, often seeking help from her grandson to } \\
\text { do so. }\end{array}$ & $\begin{array}{l}\text { Only a little } \\
\text { confident }\end{array}$ \\
\hline P3 & 71 & Female & $\begin{array}{l}\text { Some college, } \\
\text { no degree }\end{array}$ & $\begin{array}{l}\text { Smartphone, primarily for phone calls but once } \\
\text { every few days to find information, read news, } \\
\text { or check email. Also owned a computer but di } \\
\text { not use it at the time of study since it was slow } \\
\text { and needed repair. Previously used the computer } \\
\text { to play games. }\end{array}$ & $\begin{array}{l}\text { Only a little } \\
\text { confident }\end{array}$ \\
\hline P4 & 65 & Female & $\begin{array}{l}\text { Some college, } \\
\text { no degree }\end{array}$ & $\begin{array}{l}\text { None, but previously had a computer and used it } \\
\text { once a week for Facebook or online information. }\end{array}$ & $\begin{array}{l}\text { Only a little } \\
\text { confident }\end{array}$ \\
\hline P5 & 71 & Female & High school & $\begin{array}{l}\text { Owns a computer that is not functioning; } \\
\text { previously used it once every few days for } \\
\text { games, social media, and finding information, } \\
\text { often with help from her daughter. }\end{array}$ & $\begin{array}{l}\text { Not at all } \\
\text { confident }\end{array}$ \\
\hline P6 & 72 & Female & $\begin{array}{l}\text { Less than high } \\
\text { school diploma }\end{array}$ & $\begin{array}{l}\text { Uses a smartphone (her daughter's old phone) } \\
\text { for making and receiving calls only. }\end{array}$ & $\begin{array}{l}\text { Somewhat } \\
\text { confident }\end{array}$ \\
\hline P7 & 83 & Female & High school & $\begin{array}{l}\text { None, but previously had a computer set up by } \\
\text { her daughter to check email once every few days. }\end{array}$ & $\begin{array}{l}\text { Not at all } \\
\text { confident }\end{array}$ \\
\hline
\end{tabular}

in their own homes. All participants lived alone except for P6, who lived with her son. Participants were asked to avoid use of the Echo Dot by others, and P6 reported that her son did not interact with the device during the study period (P6's visiting grandson did use the device briefly, and we removed those usage logs data from our analysis). More than half of the participants had a high school degree as their highest level of education. Participants were also asked to rate their confidence in using computing devices on a 4-point scale: "very confident," "somewhat confident," "only a little confident," or "not at all confident."

\subsection{Procedure}

The study procedure consisted of a 3-week field deployment that included an initial interview and device setup session, two interim interviews, and a final interview. Daily automated calls also collected diary entries from participants. Interviews were video recorded, diary entries were audio recorded, and usage logs were extracted from the Amazon Alexa app on the paired Fire tablet.

3.2.1 Initial Interview and Device Setup. This first session consisted of a 90-120 minutes semistructured interview followed by device setup and a tutorial on how to use the device. The interview began with demographic questions along with questions on participants' internet usage, confidence of using technology and perceived quality of life. To provide an overview of smart speaker capabilities, we showed a short video demonstrating some smart speaker capabilities. This video was created by the research team and included clips extracted from popular smart speaker commercials (e.g., Amazon Echo commercial). Each clip showed a user interacting with the smart speaker to illustrate a device capability, such as answering user questions, creating/adding items to shopping list, reading the news, providing information on weather and traffic, and turning on 
lights. This introduction was followed by semi-structured questions to gauge initial perceptions of the smart speaker, including questions on initial thoughts about using a voice-based technology, desire to use such a device, and perceived usefulness. We then set up the Amazon Echo Dot and paired Fire tablet, connecting the Dot and the tablet to Wi-Fi and to a new Amazon account that we created for participants to use over the course of the study; these accounts used Gmail email addresses also created by us. A music subscription service was also added to their Amazon account so that they could access free music using their device for the duration of the study. The Echo Dot device was placed at each participant's preferred location in their home. All participants placed the device in their living room and did not move it during the course of the study (although they were allowed to change the location, if desired).

Following setup, the researcher walked participants through an introductory tutorial that covered basic device capabilities, including setting alarms, reminders and timers, creating shopping and to-do lists, playing music, asking a joke, asking questions, and having unstructured conversation with the device. After demonstrating each device feature, we asked participants their thoughts about the feature and how they performed that particular activity (e.g., setting reminder) currently (if applicable). The Alexa app on the tablet was briefly explained so that participants could delete the lists they created (a feature not supported by voice). Participants were provided with a printed list of common actions supported by the device, including those described above as well listening to the news, radio, and podcasts, and engaging in unstructured conversation with Alexa (e.g., “Alexa, how are you doing?”), including using the built-in chat app, (“Alexa, I want to chat”). While the initial device setup was done by a researcher, a sheet was also provided on how to re-connect the device to $\mathrm{Wi}-\mathrm{Fi}$ in case it got disconnected. The researcher was also available to provide support via phone. Only one participant called over the course of the study for help reconnecting her device after it had lost connection.

3.2.2 Weekly Interim Interviews. Interim semi-structured interviews were conducted at the end of the first and second weeks. These interviews took 30-75 minutes and covered questions specific to participant's experience usage of the device including benefits/challenges/concerns of using Alexa, new information found about Alexa, change in placement of the device over that past week, in addition to the probes from the daily diary calls (described below). Additionally, during the first weekly interview, at the end of this semi-structured interview protocol, participants were introduced to third-party applications on the Echo Device-that is, Alexa "skills." To provide a baseline set of third-party skills for all participants, we identified 10 popular skills across a range of categories by reviewing technical articles recommending skills [27, 28, 44, 55, 86]. We enabled the following 10 skills on each participant's device: games (feopardy, Akinator, Magic Door), information finding (This Day in History, Kayak), relaxation (Sleep Sounds, Meditation Timer), food and drinks (Allrecipes, Bartender) and one news skill, chosen by participants (e.g., CNN, NPR, Fox News). Participants were also given the opportunity to browse the Alexa app on the Fire tablet and select other skills to enable. Finally, we enabled the Skill Finder skill to participants to independently search for skills by voice.

3.2.3 Final Interview. A 75-90 minute in-person interview at the end of the third week covered overall experience with the Dot, the connected tablet, and general perceptions about conversational voice interaction. We asked questions related to the usefulness, willingness to use, challenges, privacy, and security concerns of using voice assistants, along with questions on comparison of Echo Dot with traditional technology devices and changes in perception of digital technology. As participants kept the devices as compensation, we helped participants connect the device to their existing Amazon accounts or by creating an Amazon account using their personal email addresses. 
3.2.4 Diary Entries via Phone. In addition to the in-person sessions described above, participants were called by an automated system (CallFire) every evening at a pre-agreed upon time to share their experience on that day. We used these daily calls to address the problem that participants may forget specific instances of device usage if only asked once per week. The responses from these daily phone calls were also used to probe for more in-depth responses during weekly interviews. We chose to use automated system rather than calling ourselves to reduce interference by our research team (i.e., to reduce a sense of obligation for the participant to engage with the device to satisfy the researcher). If participants indicated that they had used the device that day, the automated system asked about the approximate number of uses, activities, examples of useful, meaningful or enjoyable uses, and examples where the device was not able to do something the participant wanted. Participants received the daily calls on 18 of 22 days (including start and end days of the 3-week period), which excluded the four interview days. Although we did not enforce the request to respond to the daily calls (to minimize the interference), participants consistently responded, dictating diary entries for on average 16.0 of the 18 calls $(S D=2.0)$. The total duration of recorded calls was 165 minutes, with the average call being 1 minute 28 seconds $(S D=30 \mathrm{~s})$.

3.2.5 Usage Logs. Usage logs containing the commands spoken by participants (and registered by Alexa) were extracted from the Alexa app. All participants consented to this procedure. These logs indicate what the user said to Alexa, but not how the device responded. Due to privacy concerns, we did not extract the voice clips of the spoken commands available on the app.

\subsection{Data and Analysis}

All in-person interviews and diary entries via phone call were transcribed and qualitatively analyzed. For analysis, we used a thematic coding approach that included both inductive and deductive codes [9]. Deductive codes were informed by related work (e.g., on smart homes, privacy), while inductive codes were created based on the data. To form the codebook, one researcher read through the transcribed interviews and initially coded them, subsequently merging the initial codes into groups and emergent categories. Four additional interview transcripts (one initial and three weekly interviews) were then randomly selected and coded by the researcher, and a second reviewer external to the research team performed a peer-review on the coded transcript, marking any disagreements. Finally, both the coder and the peer reviewer discussed and resolved disagreements through consensus, updating the codebook as needed. The final codebook contained 23 primary codes related to device usage, specific use-cases (e.g., information finding), initial thoughts about the technology and change in perceptions with time, comparison with previously used technologies, challenges and limitations, privacy and security, and social context of use.

For the usage logs, after excluding commands spoken during researcher visits (e.g., the device tutorial), 4,150 commands remained across all participants. This set included 2,821 primary commands (e.g., "Alexa,...") and 1,329 in-app commands that occurred once the user was within the in-built chat application or a skill (e.g., answering questions within the Jeopardy game).

To understand general patterns of use and because third-party skills enabled varied by participant, we analyzed only the primary commands after removing instances of just the wake-up word "Alexa" on its own ( $24 \%$ of $4,150, N=998)$, commands telling Alexa to "stop" (3.9\%, $N=163)$, "text not available" and "unknown" logs automatically flagged by Amazon in the app $(10.5 \%, N=437)$, and commands that could not be comprehended by researchers due to speech recognition errors $(4.2 \%, N=174)$ (e.g., "do wine half a tesla minute"). Although the exact reason for the automatically flagged "text not available and "unknown" logs is unknown, possibilities include the device being unable to parse the recognized text and accidental triggering of the device. 
The final dataset contained 1,049 primary commands. We coded each primary command with its purpose (e.g., finding information, checking weather). Three research team members were involved in forming the initial codebook, which was validated by using a multi-step coding process [31]. Two researchers independently coded 40 commands selected from two random participants (20 sequential commands each) and discussed disagreements to update code definitions. After a second round of 60 commands from two participants, Cohen's kappa for the final three primary codes (purpose, command repetition, and complex commands) was 0.87 ( $S D=0.15$, range $=0.7-$ 1.0). One researcher coded the remaining commands.

\section{FINDINGS}

We describe overall usage and changes over time, some of the purposes for which the technology was used, current limitations of voice-only interfaces for our target population and the privacy and security aspects of this technology.

\subsection{Overall Usage}

Below, we describe participants' initial perceptions and usage of Alexa and how it changed with time, followed by the overall device usage and the specific use cases of information finding and supporting memory.

4.1.1 Initial Attitudes and Changes Over Time. Five of the seven participants had heard about voice-controlled smart speakers through television commercials and had very limited knowledge about the Echo's functionality. The other two participants (P4 and P6) had some idea about the device's basic capabilities (e.g., playing music, checking the weather, answering questions) from people they knew using a similar device. In the beginning, P1 and P5 were concerned about being able to use a new technology. One of them (P5) requested a demonstration of the device before signing up for the study to ensure that it was not too complex for her to use.

When asked at the end of the study to reflect on their initial experience, participants reported varying initial concerns. P3 at first said she "felt dumb talking to this machine," and P4 "was a little hesitant" (though with time both became comfortable using it). In the initial interview, two participants (P1, P5) were concerned about remembering the wake-up word "Alexa." This fear seemed well founded, as the majority of the participants (4/7) used an incorrect word ("Alexis") during the device setup session. Two of them learned to use the correct keyword in the first week, but we observed the other two participants continuing to say "Alexis" even during the second- and third-week interviews. When asked why she had used this term, P6 explained that switching the terms was unintentional:

I made a mistake and said, "Alexis” ... there's no reason. I just said it wrong, that's all... Just like I might holler at somebody, and just happen to call 'em by the wrong name, not thinking. [P6]

For one participant (P1), the perceived concern of using a voice-based technology at home changed over time with sustained use. P1 was initially concerned about being startled or scared by hearing a voice in his house: "I hope it don't scare me the first time. If I tell it to do something, you know how you sleep and a voice come in and [be]cause it's come on in a voice, right, like a person talking to you." However, he did not report experiencing this issue in the following weeks. Additionally, initially he thought that using the device to listen to music "might wear up quick," but was still using it for music in the second and third weeks, saying "I really got comfortable with it." 


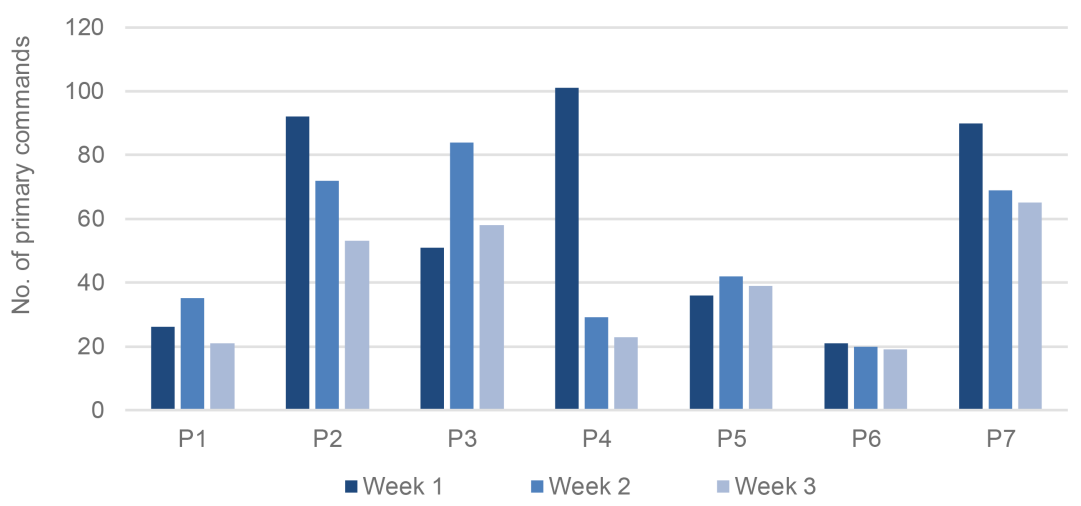

Fig. 1. Week-specific device usage for participants (based on the filtered primary commands dataset), showing some participants' usage decreasing over time while others' usage remained stable.

At the final interview, all participants reported that they found the device easy to use, their confidence in using a voice-based device had increased, and they were willing to keep using the device. For example, P3 said: "I wasn't confident initially, like I said, because it was new, but now I'm more familiar with it, so I feel more ease with it." For P7, the novelty of using the device had worn off: "At first I was more excited because it was new and now I'm comfortable. I'm still going to use it but there's no edginess"; however, her willingness to use the device had increased: "more willing now I think because I'm more familiar."

4.1.2 Device Usage. Device usage details based on analyzing the 1,049 coded conversational logs from the Alexa app are shown in Table 3. Drawing on the usage log data, here we describe how participants used the different device features. The most common tasks were finding information (34.9\%), unstructured conversations with Alexa (i.e., appearing to be primarily social, such as thank you, good morning) (13.6\%), playing music (13.3\%), and checking the weather (9.8\%). Additionally, $4.1 \%$ of commands were coded as "other," which includes volume control commands, commands to pause Alexa, repeat a previous statement (said by Alexa), to call someone, control TV, lights, and so on. These percentages include commands that were an exact repetition or slight rephrasing (e.g., "what is the time" vs. "what time is it") of the previous command in the given Alexa log $(N=157,15.0 \%)$. Of these repeated commands, slightly more than half $(54.1 \%$ of the 157$)$ were exact repetitions, whereas $45.9 \%$ included some modification. We retained these repeated commands because the logs do not contain precise timestamps, making it impossible to determine whether the participant repeated the command due to an unexpected response from the system, or because they simply wanted to repeat the action (e.g., checking the time again a few minutes later).

In terms of usage over time Figure 1 indicates that the usage dropped-off for some participants but not for others. In Figure 1, it appears that for the participants who had high device usage in the first week (P2, P4, P7), there was a reduction in use in the subsequent weeks. On the other hand, for the participants with relatively low device use in the first week (P1, P5, P6), the usage in the subsequent weeks did not vary much. P3 appears to be an exception to this latter trend as her device usage increased in the second week but decreased in the third week.

The descriptive statistics for week-specific usage is shown in Table 2. A non-parametric Friedman test was conducted to see if there was a difference in the usage (number of commands used) based on the single within-subjects factor of week (i.e., week 1 , week 2 , week 3 ). There was a statistically significant main effect of week, confirming the drop-off in usage over time that is seen in Table $2\left(\chi^{2}(3)=15.86, \mathrm{p}<0.05\right)$. 
Table 2. Descriptive Statistics of Device Usage

\begin{tabular}{|l|c|c|c|}
\hline Average Commands & Mean (SD) & Median & Interquartile Range (IQR: Q3-Q1) \\
\hline Week 1 & $59.57(34.00)$ & 51 & $92-26$ \\
\hline Week 2 & $50.14(24.60)$ & 42 & $72-29$ \\
\hline Week 3 & $39.71(19.19)$ & 39 & $58-21$ \\
\hline
\end{tabular}

Table 3. Overall Device Usage

\begin{tabular}{|l|c|l|}
\hline Specific Use & $\%$ & \multicolumn{1}{|c|}{ Used by } \\
\hline Finding information & 34.9 & All \\
\hline Unstructured conversation & 13.6 & All \\
\hline Playing music & 13.3 & All but P2 \\
\hline Skill-specific commands & 10.5 & All but P1 \\
\hline Weather & 9.8 & All \\
\hline Grocery list & 4.5 & All but P1 \\
\hline Time & 3.4 & All but P2 \\
\hline Joke & 2.8 & All but P1 \& P4 \\
\hline Reminder & 2.5 & All but P5 \\
\hline News & 1.6 & P2, P3, P5, P7 \\
\hline Story & 1.3 & P2, P7 \\
\hline Alarm & 0.9 & P1, P2, P3, P6 \\
\hline Radio & 0.5 & P1, P2, P3, P7 \\
\hline Calculation & 0.5 & P5, P7 \\
\hline Timer & 0.4 & P3, P6 \\
\hline
\end{tabular}

Note: \% refers to the percentage of primary commands $(\mathrm{N}=1,049)$ categorized under each specific use.

Table 4. The Most Common Uses of the Device across the 3 Weeks of the Study

\begin{tabular}{|l|c|c|c|}
\hline Percentage Use for the Specific Week & $\mathrm{W} 1(N=418)$ & $\mathrm{W} 2(N=351)$ & $\mathrm{W} 3(N=280)$ \\
\hline Finding information & $34.9(n=146)$ & $33.9(n=119)$ & $36.1(n=109)$ \\
\hline Unstructured conversation & $11.5(n=48)$ & $15.7(n=55)$ & $14.3(n=40)$ \\
\hline Playing music & $23.9(n=100)$ & $8.8(n=31)$ & $2.9(n=8)$ \\
\hline Skill-specific commands & $0.2(n=1)$ & $20.2(n=71)$ & $13.6(n=38)$ \\
\hline Checking weather & $8.4(n=35)$ & $10.5(n=37)$ & $11.1(n=31)$ \\
\hline Grocery list & $2.9(n=12)$ & $3.4(n=12)$ & $8.2(n=23)$ \\
\hline
\end{tabular}

Note: Third-party skills were only introduced in Week 2.

The features most frequently used over the 3-week period are shown in Table 4. Playing music, which was a more common use in the first week (23.9\%), sharply reduced to $2.9 \%$ by the third week, despite the easy access to playing free music through a linked subscription service. On the other hand, using the device to check the weather or create grocery lists increased by the third week. Below we describe the changes in use of some features, e.g., music, reminders and grocery lists (in the "supporting memory" section below), qualified by interview findings. Though our data does not include details on why participants changed their usage of some of the other categories, it appears that at least some of the initial usage of music was due to curiosity and the novelty of 
Table 5. Categories of Information Searched for by Participants (\% of 366 Commands)

\begin{tabular}{|l|c|l|c|}
\hline Category & $\%$ & \multicolumn{1}{|c|}{ Category } & $\%$ \\
\hline Health related & 16.1 & Entertainment & 3.8 \\
\hline Famous personalities & 13.1 & Special days & 3.3 \\
\hline History & 10.9 & Culture specific & 3.3 \\
\hline Local information & 9.3 & Meaning of words & 2.5 \\
\hline Food and drink & 9.3 & Specific places & 4.4 \\
\hline Alexa related & 6.0 & Math conversions & 1.6 \\
\hline Spelling & 4.1 & Technology related & 1.4 \\
\hline Travel & 3.8 & Miscellaneous questions & 18.3 \\
\hline
\end{tabular}

using Alexa for playing specific songs: P1 described in the second weekly interview how initially he had the "curiosity, to see if it could... Try asking it things and see what it'll do." Table 4 also shows consistent use of the device throughout the 3 weeks to access online information.

Although our focus is on the voice-based features of the Echo Dot, five participants (all but P1 and P5) explored using the paired tablet device for a variety of activities: games (P2, with help from student volunteers visiting the independent living facility to set up the games), the Alexa app (P3), social media (P4), music (P6), and calculations (P8).

4.1.3 Information Finding. We were interested in understanding when and how our participants used this device to access online information. Existing methods for finding information (i.e., prior to enrollment in the study) included asking someone (e.g., family members, 6/7 participants), using phone books (4/7), or using a computer (3/7). However, the participants who reported using a computer, did not do so frequently and/or independently.

P2 tended to employ her grandson's help in using her computer ("I don't normally do it on my own, my grandson will tell me.”), while P6, who did not own a computer, looked up information with help from her visiting grandson on his computer. The third person, P5, did not currently have a working computer but mentioned that she had used her computer to "try to look up [information]" in the past. Less common strategies included using phone-based services, encyclopedias, newspapers, dictionaries, cookbooks, and libraries.

Compared to these existing methods of information finding, all participants valued the ability to ask Alexa questions on a variety of topics. Participants referred to Alexa as "an invisible person with knowledge" (P3), as well as a "dictionary" (P1 and P5), "encyclopedia" (P7), and "learning device" (P6). They explained that they used the Echo for finding information for a number of reasons, including curiosity and to double check or further learn about topics that they had heard from other sources (e.g., other people or television).

The usage logs included 366 information-finding commands spanning a variety of topics (Table 5). The most frequent topic was health (16\% of 366) -an area that past research has found is commonly sought by older adults [51]. But, in addition to health-related searches, participants also sought information related to famous personalities such as celebrities or political figures (13.1\%), history (10.9\%), local information (e.g., restaurants, movie theatres, 9.3\%), and food or drinks (e.g., "Alexa, how do you make dumplings," 9.3\%).

Highlighting the importance of health information seeking in our data, all but one participant asked health-related questions over the course of the study. These were often specific to health conditions or medications (e.g., questions related to Parkinson's disease, rheumatoid arthritis, 
hepatitis, diabetes, desired blood pressure range, desired white blood cell counts), questions on specific medications (e.g., Prevnar 13, prednisone, Prozac), or approaches to manage day-to-day health occurrences (e.g., headache, foot pain). Participants used Alexa to double check information from others, and P3 used the device to check information from their doctor about ways to control diabetes besides medicine:

... some of my questions were on what other avenues you could try even if you are taking medication. And it would tell you exercise and things like that, watch your diet. So, something like that ... your doctor can tell you, but if somebody else also tell you same thing, then you know, it must be true. [P3]

Participants also found the device to be useful for finding local information. For example, P7 asked Alexa about the nearest bank, then used the information on the hours and location of the nearest branch. P3 found the nearest dentist through Alexa, then called and set an appointment, for which otherwise she would have to "go through a phonebook or ... ask other people." Similarly, for finding information about nearest movies and their timings, participants described using Alexa, instead of asking someone (P2, P6), looking in newspaper (P7) or calling the theatre (P1)-which they said they would have done if Alexa was not there.

The credibility of the information provided by Alexa was mentioned by a few participants. P7, for example, trusted the information provided by Alexa, and perceived Alexa to be more credible than asking someone. She described using the device to help settle a factual argument with her friend about a particular fact was true about a celebrity, explaining that without it:

I would have said maybe you're right. I will still believe what I'm believing but I couldn't prove... But I felt good being able to have a source that I could quote ... I told her where I got my information and she said, "Oh, okay." [P7]

Unlike P7 and her friend, P3 wanted to double check the information she received "to see if this [Alexa] would tell me the same thing that I found out on the computer" (though she reported not actually double-checking this information during the study as her computer was too slow and needed repair).

Some participants (2/7) also valued that there were no negative social repercussions from asking Alexa questions. For example, P7 said, "Alexa ... never says, 'Why are you asking me that'," while P3 reported that her grandchildren said:

"You need that [Alexa]!" That's what they told me. I said, "Why?" "Because you don't understand, and lots of times when we'd be telling you, and now you can listen"... Because they [grandchildren] get frustrated... "Well Grandma, I told you that the other day. You don't remember?" That's what they'll tell you. [P3]

P3's comment resonates with past work, which notes that older adults often seek help from family members and friends for general information [32,34,80], yet might not always be satisfied with the help received (e.g., because others have little patience with them when they ask for assistance [80]). Four participants also mentioned that since the device lowered barriers to asking questions, it spurred curiosity and led them to find information when they might not have otherwise. For example, P5 explained that if she did not have the device she might just "forget it [her question]" rather than looking it up on the computer because "I just am too slow with that [the computer]." After using Alexa, P6 and P7 realized how curious they were: 
I think I'm more curious than I realize I was. Because I look at TV so much, I'm just zoomed in on it, and I stop thinking independently about things that... And so when I cut the TV off, I know that I can ask questions now to Alexa. [P6]

You do find that you're thinking more and you're thinking about going to the next step instead of just having the question in your head and letting it go, you say, "I wonder if the device can answer this question?" [P7]

Similarly, for P3, although she was curious about her culture, she "wouldn't have picked up a book and went to look up this information on black history myself. So, having that for me, it made me want to learn and ask questions."

4.1.4 Supporting Memory. We also wanted to understand how the device supports memoryrelated tasks, as some participants (3/7) reported having at least some challenges with memory that affected everyday activities. Prior to using Alexa, all participants reported using handwritten notes and paper calendars for reminders, while three participants also mentioned enlisting family or friends to call them with important reminders (e.g., appointments).

Device features such as setting reminders, alarms, timers and creating lists (to-do list, shopping list) were initially perceived to be useful by all participants. However, the recorded usage of these features was less than might be indicated by this initial enthusiasm. For features such as reminders and alarms, usage decreased with time. Although reminders were used at least once by most participants (all but P5), only 26 commands (2.5\%) in usage logs involved setting reminders, most of which (18/26) were in the first week. The most common use of device features that could support memory was to create grocery lists (used by all but P1). The command log analysis indicates 47 commands (4.5\%) related to grocery lists. Interestingly, the use of grocery lists increased with time, with about half of the usage being in the third week (23/47), also indicated in Table 3. During the second weekly interview, P4 described how using shopping list had developed into a habit, possibly accounting for the increased usage for grocery lists in the usage logs:

I've been using it for my shopping list, which is kinda nice because every time I think of something, I don't have to... If I think of something, I can tell it [Alexa] and I'm not writing it down, so I will write it down before I go to the grocery store. $[\mathrm{P} 4]$

For the reminders and alarms, the interviews provided additional context for the limited use of these features. Though three participants described cases where they would have forgotten to do something if they had not had the Echo (e.g., through a reminder to call someone the next day), most participants (5/7) did not find the reminders or alarms reliable. Two of these participants were concerned about remembering to set a reminder or an alarm on the device. For example, P5 described concerns related to relying on her own self as she was experiencing changes with short-term memory that led her to ask her daughter to call her with reminders. She felt she could have benefited by Alexa's reminders but forgot to do so:

I do have a reminder about going to the doctor and I would have needed a reminder yesterday to pay my rent... I didn't think about it... There was a lot of things that I probably could have done. [P5]

However, she was positive about letting others (her daughters and her doctor) set reminders remotely on her device, highlighting the use of collaborative remembering as a strategy to support memory. 
Two participants who used the reminder feature mentioned using Alexa as a backup to their existing methods (e.g., paper notes, calendars). P1 described his discomfort relying completely on Alexa to remind him about important medications as hesitance to put his "life in the hands of a machine." He said:

Because certain things are too important to rely on that [Echo Dot], you gotta have a backup. In other words, if I gotta be at a meeting, an interview at 9 o'clock, and I really want that job... I'm not gonna rely on Alexa. I'm gonna ask her to [set an alarm]. But I'm gonna also set all kinds of alarms. I'm gonna have friends and family to call and make sure I'm up to get there. [P1]

While both P1 and P5 had reliability concerns, it is important to note that the latter voiced concern related to her own abilities-specifically, forgetfulness. P1, however, voiced concerns due to distrust that technology could be relied on for important tasks. Other participants provided context for this distrust, explaining that use of Alexa for reminders meant they needed to be physically present to hear reminders/alarms. Others noted concerns that relate to dependence on digital technology more broadly, noting hesitance to rely on potentially unstable infrastructure (e.g., electricity, internet connection. Three participants, however, did note benefits regarding the ability to set reminders by voice to avoid losing handwritten paper notes or due to the dexterity required to write notes. Similar to findings about looking up information, two participants (P3, P7) valued the ability to repeatedly ask Alexa about the same thing and saw this as related to supporting memory.

Participants identified desired features for supporting memory that were not currently available, such as storing notes, memos, addresses or phones number and later asking Alexa to retrieve them (P3, P5) and connecting with social media (Facebook) to receive birthday reminders (P4). Two participants (P3, P6) merged reminders and shopping lists, asking the device to remind them of the things they needed to get: "remind me at two o'clock tomorrow afternoon that I need to get bread and water at the grocery store."

\subsection{Comparison with Previously Used Technologies}

We were particularly interested in how participants, who were infrequent technology users, felt about the Echo Dot compared to other devices. All participants positively compared the Echo Dot to previously used devices such as computers and smartphones. They mentioned feeling more confident using voice-based technology and mentioned the ease of learning and using this technology. For example, P7, who previously used the computer with help from her daughter, found the device easy enough to use independently: "I don't need anybody to help me with this device. I don't need anybody to walk me through what to say next because it's so simple." She highlighted the approachability of this technology, saying, "For a senior [who] just started out if they had no knowledge of either... I would say skip the laptop and go with the device if you want information."

The majority of participants (5/7) found the device to be faster and involve fewer steps than a traditional computing device, while three participants valued its hand-free nature. Two participants also appreciated that voice-based interaction had accessibility benefits: not needing to adjust text size (P7), and not needing to type or write (P2, who had arthritis). Several participants said that the ease of use resulted in an increased willingness to learn about technology. For example, at the end of third week, P2, who said that she did not have "the patience to learn a lot of stuff on the computer," but was interested in learning more about the Alexa:

I wanna know more or what else can I get into with that, because it is so easier and more simple for seniors than trying to type on a computer, go here and go there and lose the passwords and all [that]... [P2] 
For P5, the overall confidence of using other kinds of technology also increased:

This [Echo Dot] has made me a little bit feel that I could learn some new technology. And at first I was nervous. And now I'm not... I feel like I've learned how to use that. So that's an accomplishment for me because it's technology and I just like it. In the beginning, I wasn't real confident, but I thought in time as I used it more, I'll be more confident and that's how it went. [P5]

The Echo Dot altered some participants' technology use routines. P2, who had been using her computer about once a week before the study, did not use it at all during the 3 weeks of study. Three participants used Alexa for checking the weather instead of turning the TV on. Alexa was seen as an addition to existing entertainment sources (e.g., TV, radio). P3 used the TV less than before and stopped using her radio, instead playing music on her Echo Dot. Along with reduced usage of some devices at home, using the Dot led to an increased motivation for using other voicebased computing devices. For example, P7 wanted to buy a smartphone to have a portable voice assistant:

I had thought about it, but I became more curious about it, I think after I got Alexa... when I saw my daughter speak to her phone, and I'm speaking here. I said oh, okay. So, with a smart phone you can; if at home I will probably use Alexa, but if I'm in the street, the smart phone would really work great. [P7]

Even though remote voice-only interaction was valued, in certain instances participants preferred using a visual interface or a traditional computing device, such as for sending and receiving emails (3/7) and playing games (3/7), suggesting intelligent voice assistants with visual interfaces (e.g., Echo Show), might be perceived differently by these participants. For example, P3 said:

You get less time to think on this than you would if you were playing on a tablet.

She kept telling me, "You only have 6 seconds left" [jeopardy game]. I am thinking, and my time is running out... I think I enjoy seeing versus talking. [P3]

\subsection{Challenges and Barriers to Use}

Most commonly, participants reported issues due to voice recognition and the device not being able to answer a question. In both cases, the voice assistant (Alexa) gives the same output feedback. In the interviews, participants mentioned often repeating their command a few times $(\sim 2-3)$ before giving up. Instances where the device forced participants to rely on the paired computing device (e.g., using the tablet to delete items off the grocery list) were also problematic because participants were not all comfortable with using the tablet. Some participants $(N=4)$ reported problems with the device timing out after using the wake word due to a delay in speaking their complete command. This frustrated one participant, P5, as she had to go back and "do the whole thing over, and then you're worried and you're trying to do it really fast that it is gonna shut off again." Much of these challenges identified by participants largely reflect past work [61], e.g., issues with voice recognition, the device timing out before speech input is complete, and dependency on a paired computing device. In addition to those challenges, participants experienced difficulty in remembering specific voice commands, discovering device features using voice commands, and had a tendency toward using complex commands. Below we describe these specific issues related to using voice commands.

Remembering specific voice commands. Some skills and/or device features (e.g., reminders) require the user to say specific keywords. Five participants experienced difficulty with in remembering these keywords either to invoke the feature/app itself or to navigate within the feature/app 
to achieve specific goals. For example, $\mathrm{P} 2$ was unable to use a third-party app (Bible skill), which she had activated in presence of the researcher during the introduction of the Alexa skills. Compounding the problem of remembering keywords, applications that used navigational commands (e.g., "more information" or "next") were particularly challenging. These commands are inconsistent across built-in device features and third-party apps. P7, who learned to use a voice navigation command for one app ("more information" in the Allrecipes skill) tried applying it to other tasks and was unsuccessful ("more information m. and t. bank," "more information on podcast.")

Another confusing aspect of voice-based interaction was related to reminders, timers, and alarms. The output of all these features is to alert the user at some time in the future, but each function requires specific input keywords. For example, the only time P5 tried to set a reminder, she did not use the required keyword (remind/reminder) and said, "Alexa, I would like you to tell me when it's 6:00," which was not recognized by the device as a reminder (at least at the time of the study). Further, she wanted to set the reminder a day before the actual appointment by using a simple voice command (remind me today that I have an appointment tomorrow at 7.40 AM, doctors), and to delete reminders by voice (cancel my appointment on March 31st) but was unable to do so. Although P5 noted concerns around forgetting to set reminders as a primary reason for not using the feature, she experienced the need to use specific keywords as quite irritating: "I would get very frustrated with that if it wasn't gonna tell me and it said different timer or whatever and I was like I'm not going to put these [reminders] in there."

Complex or compound commands. Participants attempted complex or compound commands that were not recognized. P3 wanted the device to perform complex tasks in a single voice command, such as setting a reminder to trigger the news skill (I want Fox 5 news to come up at 6 o'clock) or set a timer to stop the radio at a certain time (if I could ask it to tune into that station and keep it on continually, until a certain time.) As shown in the usage logs, all participants tried complex commands that were not understood by the system (total of $N=25$ across participants), such as: "Alexa my foot hurts what can i use to make it better" (P1) and "Alexa, stop music for a minute and you also give me the temperature" (P4).

Discoverability of features using voice commands. Discoverability was an issue for three participants, all of whom also mentioned that a print manual would be helpful for learning (an approach examined in previous work [29]). Two participants who wanted to use the device for emergency purposes-to call emergency services or to some specific people-were not able to find the related skills, highlighting the difficulty and/or the lack of user effort to find new device features (in-built features or third-party skills). According to the logs, $6 \%(N=22)$ of all information-finding questions were aimed at learning about device capabilities or third-party skills: "what are some of the things you can do?" (P3), "can you add numbers for me" (P7) and "can you save songs that I like" (P4). This indicates that these participants may have found it intuitive to use the conversational interface for finding more information about device capabilities.

Related to these above issues with command formulation, over time, two participants described how they had learned how to speak to the device by the end of the third week. P2 mentioned framing her "words differently" and P7 remembered "to not be wordy, go right to the topic."

At first I was just talking like I was talking to you, but I understand that the way you ask a question determines whether or not it understands what you're trying, information you're trying to get. (P7)

Needing to adhere to structured and ordered commands is at odds with natural conversation, a finding that aligns with past work on limitations in the conversational abilities of voice assistants [59]. This disconnect between what Alexa appears to be able to do (engage in natural 
conversation) and its actual capabilities may have led to initial frustration and, for some, not using certain functions such as reminders.

\section{PRIVACY AND SECURITY}

All participants, except for P3, had no concerns regarding conversations being recorded by the device and the "always-on" feature of Alexa, which has often been identified as primary privacy concerns of voice-based personal assistants $[24,52,90]$. The commonly cited reason (4/6 participants) for having no such concerns was the nature of conversations they had with Alexa and in their home environment. For example, P8 said "I'm not doing anything that or saying anything that I figure is so sensitive or so secretive that I would have to not let it be heard." Although P1 and P8 did not have any concerns regarding the device recording their conversations, they were "curious" to know "when" and "why" Alexa is collecting data/recording. On the other hand, one participant (P3) had privacy concerns due to the device recording conversations: "It's never gonna be private." Further, as a security concern, she did not want to use her Echo Dot for any financially sensitive transactions such as voice shopping on Amazon.

Beyond the "always-on" privacy aspect, we also observed concerns due to the placement of a voice-based device in the home environment. Use of Alexa by other people at home, such as visitors, was noted as a concern by three participants. P3, P5 did not want other people to use the device in their absence. P6 further described Alexa's use by her mischievous visiting grandson who said Alexa to call the police, "Alexis, call the police, come get my grandma." Such commands could potentially have serious consequences, reinforcing her concern of authorized use of Alexa by someone in her house. P3 who had similar concerns of other people visiting her and said she wanted the Echo Dot to be programmed to only respond to her voice, as in that case, "You don't have to worry about other people messing with your stuff. That's my personal device."

Similarly, talking to the device in presence of other people was also a concern. Two participants (P5, P6) were concerned of speaking with Alexa in presence of other people. For P5, it was her shyness for which she "wouldn't be talking to that [Alexa] and have somebody else," whereas for P6, it was the concern of what other people would think about talking to a machine and she did not want "people think I'm crazy." This aligns and adds to previous findings of location-based privacy concerns of using voice technologies [20]. In addition to public spaces [20], we found that the private in-home environment can also lead to privacy concerns.

\section{DISCUSSION}

This study involved seven older adults who did not use computing technology regularly. We examined how participants use a voice-based interface embodied in smart speakers in their homes over the course of a 3-week period. Our findings demonstrate the potential of conversational voice-based interface to enable digital interaction and access to online information for these users. Aligning and extending previous work with older adults in general [67] we found that voice-based interfaces can increase the overall confidence of using digital technology for older adults who do not use computing devices regularly. As initially identified by Pradhan et al. [61], our findings confirm that voice-based interfaces can provide easy access to digital technology for older adults and for digitally inactive users. Similar to previous findings, issues due to device timing out $[61,83]$ errors due to speech recognition/mistranscription, and lack of conversational nature [59] and the need to remember specific keywords [61] is challenging for older adults. To this literature, we add that using smart speaker-based personal assistants for reminding purposes (e.g., reminder, timer, alarm) may face additional challenges with this population. Below, we discuss the implications for using a voice-based assistant for information finding and supporting memory, 
design considerations for building voice-only applications, and how our findings challenge traditional perceptions about digitally inactive users and can extend to other user groups.

\subsection{Health Information Seeking on Voice-Only Interfaces}

Participants used the device to search for a variety of information, with health and medical questions being the most common. Much previous work has explored older adults' online health information seeking behavior using traditional visual interfaces to search for disease symptoms, prognosis and treatment options [47]. It is unsurprising, therefore, that participants would use the voice assistant (Alexa) for similar purposes. Pertaining to credibility of information obtained in general, our findings indicate that some participants would trust any information received from the voice assistant, whereas others wanted to verify the information received from the voice assistant with a secondary source but did not actually do that during the study, citing lack of resources as the reason.

As voice-based interfaces become more widespread, it is important to consider differences that exist between search in this interface (purely non-visual) and traditional computer-based searches for all populations and how they impact the ways that users perceive and make judgement of health-related information received from such VUIs. Discerning between sources of health information is a key component of health literacy and particularly important for digital health literacy [69]. Unlike visual search interfaces, for a particular query, VUIs do not provide multiple information sources or contextual data (including page layouts, popups, advertisements, etc.) associated with a webpage-essential elements for discerning trustworthiness of information on a website $[15,74]$. Research should examine how VUIs might improve in domains such as health, where credibility is essential. For users who do not use the internet frequently (our participants) or are new to using the internet might lack "credibility judgement skills" and thus experience challenges in assessing the quality of online information. This has been confirmed in prior work where older adults' health information seeking behavior indicates that although contextual cues (e.g., page look, source identity) are important information credibility indicators, older adults are less likely to pay attention to these cues than younger adults [41]. Thus, even though some voice assistants currently reveal the source of information (e.g., Mayo Clinic, WebMD), as indicated by prior work, these sources often go unlooked or unheard (as in the case of VUIs) and hence might not be enough to address the issue of information credibility.

A straightforward approach to this issue could be to steer people toward better sources (e.g., away from WebMD.com, a commonly used for-profit company with sponsored content, toward MedlinePlus, a site produced by the National Library of Medicine). However, a more reliable approach would be to include various features of a webpage-a technique that has been widely explored for GUIs. For GUIs, augmenting search results and webpages using visualization techniques to show various metadata including website's overall popularity, popularity among domain experts, number of ads, number of awards received by website, e.g., HON award for health websites, have been successful in improving the accuracy of the end user's credibility assessment of online information [71]. Although showing visualizations is not an option for purely non-visual VUIs, using a similar range of webpage features to assess information credibility should be further explored for VUIs. For example, crowdsourcing paired with machine learning techniques can be explored to assess information credibility on health-related webpages, giving each website credibility scores, which can be returned to the user along with the query result.

\subsection{Voice Assistants for Supporting Memory}

Researchers have actively investigated reminder systems for older adults (e.g., exploring synthetic speech reminders [87], multimodal reminder systems [84]), particularly for people with 
cognitive impairment (e.g., using unsolicited prompts to remind users to set reminder on smartphone apps [33]). Although perceived as useful initially by all participants in our study, the scarce use of device features to support memory and participant feedback suggests that voice-based reminder systems, as currently instantiated in smart speakers, need to be improved to be useful. One reason participants did not make use of reminders was because they worried about system failure, for example, smart speakers not functioning due to power outage or internet failure. Past work with older adults has investigated physical interfaces that connect to digital GUI systems: for example, paper checks that link to online payment systems through the use of digital pens [57, 81], thereby linking the design metaphor (i.e., an HCI design concept which "communicates to users what a computer application could do by linking it to something already familiar to the user" [35]) to the actual physical object with which users are comfortable. Researchers could explore designing with similar conceptual metaphorical linkages on VUI systems as well. For example, designers could incorporate voice-based systems as backups to interfaces people rely on, e.g., by connecting digital reminders to paper calendars through digital pens or other technologies.

For concerns related to the user forgetting to set reminders or alarms, collaborative remembering strategies, i.e., where more than one person is responsible for recall [10], should be further explored. Allowing options for other "trusted" people (e.g., close family member, caregiver, doctor) to set reminders and alarms remotely, could potentially help alleviate the issue. Another issue that surfaced particularly with memory support features was related to ways that requests (i.e., what command the user gives to the voice assistant) and responses (what, how the voice assistant responds back to the user) have been programmed for the voice assistant-a common issue with conversation design and VUIs [59]. All of the three memory features, alarms, reminders and timers, perform similar a response action, which is alerting the user at a specific time in the future. However, the input request (needed from the user) is different for these features, thus confusing some users, as indicated in our findings. Such issues with request and response design can lead to usability concerns, with repeated occurrences ultimately leading to non-use of such features despite initial excitement about these features. Although ongoing work in natural language processing [78] can help address these issues by better understanding the intent of the request, designers could also consider grouping similar actions together and reduce the dependency on using specific keywords in the request.

\subsection{Implications for VUI Design}

We identified several design considerations for voice-based interfaces. Since the usage log data only provided the user request and not the device response, much of our inference is based on the latter, triangulating it with our interview data. Beyond issues already identified in previous work (e.g., discoverability, speech recognition issues, device timing out before completion of commands [61]), our study suggests a need for consistent interaction across built-in device features and thirdparty applications. The difficulty in formulation of voice commands for using VUIs draws attention to the confusion possibly stemming from the GUI-based interaction model being applied to VUIs. For traditional GUIs, heuristics have focused on maintaining consistency and standards across the interface design $[50,73]$. Our findings indicate how inconsistent voice commands affect the overall learnability and usability of using the VUI. As such, ongoing work on compiling heuristics for designing VUIs [85] should also include consistency of specific keyword (or request) use as an important heuristic.

Issues related to remembering specific keywords for using certain features on the voice assistant was a challenge for many participants, highlighting concerns around the cognitive accessibility of this technology. With traditional computing devices, this issue might not arise as often, since the input options are visible to the user. VUI designers could explore visible input options 
to complement the voice only input, e.g., screen-based (e.g., Echo Show or on the paired tablet) or holographic projections of possible input requests a user can give to the voice assistant. Further, instead of expecting that users remember exact keywords, VUI designers should follow heuristics of confirming user input, i.e., if user forgets to say the "exact" keywords, the assistant should predict the input request and verify it from the user [85], and by incorporating contextual cues (e.g., location of the user, recently asked requests by the user) into the conversation design where possible.

To reduce the dependency on the paired tablet, there is an opportunity for response feedback to expand beyond predefined responses (such as "I don't understand," "Sorry, I don't know that") to help participants in troubleshooting. For example, when the system fails to perform the requested task (e.g., answer a question), it should provide more specific feedback that can aid troubleshooting (e.g., differentiating between lack of device knowledge vs. speech recognition error due to not understanding the user or due to background noise). Understanding the user's natural progress of talk during interaction with the voice assistant might enable designers to overcome some of these issues [22].

\subsection{Non-frequent vs. Frequent Digital Technology Users and VUIs}

In contrast to previous perceptions of non-frequent technology users' disinterest toward learning to use technology [49], we found that participants not only learned how to use the smart speaker within a few weeks, but also expressed interest in continuing to use the device after the study. This may be due to the voice-based interaction and absence of visual interaction elements found in traditional computing devices-often attributed as a barrier to technology adoption by novice users [46]. With the widespread popularity of voice-based technologies, researchers have begun understanding use of VUIs by technologically novice users (e.g., speech-based social media platform [63]). Our findings pertaining to increased confidence of using digital technology with voice-based interfaces, primarily due to the ease of use, adds to this thread of work. However, this body of work is still in early stages and it is important to understand the use of voice-based interfaces by users who are less motivated to use digital computing technology in general.

At the same time, it is possible that the issues infrequent technology users experienced using voice-based interfaces, such as speech misrecognition, could actually be more frustrating for older adults who are frequent users of traditional GUIs such as computers, smartphones, or tablets. These older adults already have a reliable source for accessing online information. For individuals in our study, who were not frequent users of digital computing devices, the benefits may have led to a greater tolerance for the frustrations that they experienced.

\subsection{Limitations}

One limitation of our approach is that though we studied the use of these devices over 3 weeks by "low-tech" older adults, the adoption was forced. Forced adoption was the best option available for us, since we were specifically interested in older adults with low technology use. If individuals regularly used these devices, they could not be categorized as low-tech users. Studying new device using forced adoption has been observed in HCI research, e.g., in the case of smart speakers [59], Google Glass [45], and smart sensors [39]. Since it was a forced adoption, the study was not an entirely naturalistic deployment. We provided participants with training to use the basic device and third-party skills as well as gave them a printed reference guide that we created. Participants could also contact the researchers at any point for support, and support for use could be provided during the weekly interview.

Having daily diary calls has tradeoffs. The calls enabled us to capture "in-the-moment use" in lieu of observation (as observations might influence use more than calls) and helped us to probe 
for in-depth explanations during weekly in person interviews. Yet, this may have affected the natural use of the device since participants were frequently reminded about the research study and presence of the device. To minimize this impact, we used automated calls instead of a research team member calling. Further, the longitudinal nature of the study over 3 weeks also has tradeoffs. Although it enabled us to gather an in-depth understanding of device use, the longitudinal nature of this study using a range of data sources made it practically difficult to obtain generalizable results-a limitation of using this method [36].

The specific applications participants used might have been influenced by those presented by the researchers. Moreover, recording of usage logs of participants' interaction with the device could also have created a self-consciousness affecting the nature of questions people asked to the device. Additionally, the collected logs are not fully accurate (some automatically flagged "text not available" and "unknown" in the Alexa app) and contain successively repeated commands without a timestamp, making it impossible to calculate the exact number of repeated commands accurately. Using a custom-made voice recorder (e.g., similar to [59]) might have helped eliminate these issues. Finally, the participant sample was not balanced in terms of gender, with six women and one man. All findings in this study are subject to Amazon Alexa's functionality at the time of study. There may have been updates to this technology since then.

All participants except P6 lived alone, and individuals were intentionally asked to be the sole user of the Echo Dot. Hence, one limitation of our work is that we do not analyze the social dynamics associated with the use of the voice assistant, instead focusing on individual use. Future work needs to further investigate how such voice assistants fit into older adults' social ecosystem and in-home dynamics.

\section{CONCLUSIONS}

Voice assistants embedded in smart speakers have become increasingly popular in the past few years. These offer unique affordances that have the potential to lower the barrier to technology access for certain user groups. In this study, we deployed smart speaker devices for a 3-week period in the homes of older adults who do not use computing devices every day. Throughout the 3-week period, we found that older adults consistently used the device for accessing online information. The use of the device to access health-related information as one of the most common information types warrants a need for future work to examine the concerns of information credibility when using non-visual, voice-only interfaces for this purpose. While most participants were initially excited about using memory support features such as reminders, the actual use of those features was much less than expected. Participants cited reasons such as failure of the technology and forgetting to set the reminder using the voice assistant, opening up opportunities for researchers to explore conceptual metaphorical linkages with currently used physical objects that users are comfortable with; for example, a paper-based calendar linked with the voice assistant itself could lead to increased trust and comfort of using a new technology. Our work also provides preliminary insights into how voice-based technologies can lower the barrier of technology access due to the ease of using the interface.

\section{ACKNOWLEDGMENTS}

Opinions expressed do not necessarily represent official policy of the Federal government. We would like to thank Kanika Mehta for helping with data analysis. We are also thankful to our participants for volunteering to participate, and the coordinators of nearby independent living facilities for helping recruit participants. 


\section{REFERENCES}

[1] Amr Alanwar, Bharathan Balaji, Tian Yuan, Shuo Yang, and Mani Srivastava. 2017. EchoSafe: Sonar-based verifiable interaction with intelligent digital agents. In Proceedings of the 1st ACM Workshop on the Internet of Safe Things (SafeThings'17). 38-43.

[2] Tawfiq Ammari, Jofish Kaye, Janice Y. Tsai, and Frank Bentley. 2019. Music, search, and IoT. ACM Transactions on Computer-Human Interaction 26, 3 (2019), Article 17. DOI : https://doi.org/10.1145/3311956

[3] Monica Anderson and Andrew Perrin. 2017. Technology use among seniors. Pew Research Center. Retrieved September 20, 2018 from http://www.pewinternet.org/2017/05/17/technology-use-among-seniors/.

[4] Monica Anderson and Andrew Perrin. 2017. Barriers to Adoption and Attitudes Towards Technology. Retrieved September 1, 2018 from http://www.pewinternet.org/2017/05/17/barriers-to-adoption-and-attitudestowards-technology/.

[5] Monica Anderson, Andrew Perrin, and Jlingjing Jiang. 11\% of Americans don't use the internet. Who are they? Retrieved August 18, 2018 from http://www.pewresearch.org/fact-tank/2018/03/05/some-americans-dont-use-theinternet-who-are-they/.

[6] Anne Aula. 2005. User study on older adults' use of the web and search engines. Universal Access in the Information Society 4, 1 (2005), 67-81. DOI : https://doi.org/10.1007/s10209-004-0097-7

[7] Momotaz Begum, Rosalie Wang, Rajibul Huq, and Alex Mihailidis. 2013. Performance of daily activities by older adults with dementia: The role of an assistive robot. In Proceedings of the IEEE International Conference on Rehabilitation Robotics. 1-8. DOI : https://doi.org/10.1109/ICORR.2013.6650405

[8] Diana Beirl, Nicola Yuill, and Yvonne Rogers. 2019. Using Voice Assistant Skills in Family Life. International Society of the Learning Sciences. Retrieved from https://repository.isls.org//handle/1/1750.

[9] Virginia Braun and Victoria Clarke. 2006. Using thematic analysis in psychology. Qualitative Research in Pyschology 3, 2 (2006), 77-101.

[10] R. N. Brewer, M. R. Morris, and S. E. Lindley. 2017. How to remember what to remember: Exploring possibilities for digital reminder systems. Proceedings of the ACM on Interactive, Mobile, Wearable and Ubiquitous Technologies 1, 3 (2017), Article 38. DOI : https://doi.org/10.1145/3130903

[11] Robin Brewer, Raymundo Cornejo Garcia, Tedmond Schwaba, Darren Gergle, and Anne Marie Piper. 2016. Exploring traditional phones as an e-mail interface for older adults. ACM Transactions on Accessible Computing 8, 2 (2016), Article 6. DOI : https://doi.org/10.1145/2839303

[12] Robin N. Brewer and Anne Marie Piper. 2017. xPress: Rethinking design for aging and accessibility through a voicebased online blogging community. Proceedings of the ACM on Human-Computer Interaction 1, CSCW (2017), Article 26. DOI : https://doi.org/10.1145/3139354

[13] Robin Brewer and Anne Marie Piper. 2016. "Tell It like it really is": A case of online content creation and sharing among older adult bloggers. In Proceedings of the 2016 CHI Conference on Human Factors in Computing Systems. 55295542. DOI : http://dx.doi.org/10.1145/2858036.2858379

[14] Rebecca Cherng Shiow Chang, Hsi Peng Lu, and Peishan Yang. 2018. Stereotypes or golden rules? Exploring likable voice traits of social robots as active aging companions for tech-savvy baby boomers in Taiwan. Computers in Human Behavior 84 (2018), 194-210. DOI : https://doi.org/10.1016/j.chb.2018.02.025

[15] Wonchan Choi. 2013. What makes online health information credible for older adults? In Proceedings of the CHI'13 Extended Abstracts on Human Factors in Computing Systems (CHI EA'13). 2671-2676. DOI : https://doi.org/10.1145/ 2468356.2479491

[16] Michael Crabb and Vicki L. Hanson. 2016. An analysis of age, technology usage, and cognitive characteristics within information retrieval tasks. ACM Transactions on Accessible Computing 8, 3 (2016), 10. DOI : https://doi.org/10.1145/ 2856046

[17] Sara J. Czaja, Neil Charness, Arthur D. Fisk, Christopher Hertzog, Sankaran N. Nair, Wendy A. Rogers, and Joseph Sharit. 2006. Factors predicting the use of technology: Findings from the center for research and education on aging and technology enhancement (CREATE). Psychology and Aging 21, 2 (2006), 333-352. DOI : https://doi.org/10.1037/ 0882-7974.21.2.333

[18] Gilbert Dizon. 2017. Using intelligent personal assistants for second language learning: A case study of alexa. TESOL fournal 8, 4 (2017), 811-830. DOI : https://doi.org/10.1002/tesj.353

[19] Stefania Druga, Randi Williams, Cynthia Breazeal, and Mitchel Resnick. 2017. "Hey google is it ok if i eat you?" Initial explorations in child-agent interaction. In Proceedings of the 2017 Conference on Interaction Design and Children (IDC'17). 595-600. DOI : https://doi.org/10.1145/3078072.3084330

[20] Aarthi Easwara Moorthy and Kim-Phuong L. Vu. 2015. Privacy concerns for use of voice activated personal assistant in the public space. International fournal of Human-Computer Interaction 31, 4 (2015), 307-335. DOI : https://doi.org/ $10.1080 / 10447318.2014 .986642$ 
[21] Flávio Ferreira, Nuno Almeida, Ana Filipa. Rosa, André Oliveira, António Teixeira, and José Casimiro Pereira. 2013. Multimodal and adaptable medication assistant for the elderly: A prototype for interaction and usability in smartphones. In Proceedings of the 8th Iberian Conference on Information Systems and Technologies. 1-6.

[22] Joel E. Fischer, Stuart Reeves, Martin Porcheron, and Rein Ove Sikveland. 2019. Progressivity for voice interface design. In Proceedings of the 1st International Conference on Conversational User Interfaces (CUI'19). Paper 26. DOI : https://doi.org/10.1145/3342775.3342788

[23] Thomas N. Friemel. 2016. The digital divide has grown old: Determinants of a digital divide among seniors. New Media and Society 18, 2 (2016), 313-331. DOI : https://doi.org/10.1177/1461444814538648

[24] Nathaniel Fruchter and Ilaria Liccardi. 2018. Consumer attitudes towards privacy and security in home assistants. In Proceedings of the 2018 CHI Conference Extended Abstracts on Human Factors in Computing Systems. Paper LBW050. DOI : https://doi.org/10.1145/3170427.3188448

[25] Susan L. Gatto and Sunghee H. Tak. 2008. Computer, internet, and e-mail use among older adults: Benefits and barriers. Educational Gerontology 34, 9 (2008), 800-811. DOI : https://doi.org/10.1080/03601270802243697

[26] Vinícius P. Gonçalves, Vânia P. de Almeida Neris, Sibelius Seraphini, Teresa C. M. Dias, Gustavo Pessin, Thienne Johnson, and Jó Ueyama. 2017. Providing adaptive smartphone interfaces targeted at elderly people: An approach that takes into account diversity among the elderly. Universal Access in the Information Society 16, 1 (2017), 129-149. DOI : https://doi.org/10.1007/s10209-015-0429-9

[27] Eric Griffith and Rob Marvin. 2017. The best amazon alexa skills. PCMag.com. Retrieved March 25, 2018 from https://www.pcmag.com/article/352136/the-best-amazon-alexa-skills.

[28] Matt Hanson and Jon Porter. 2018. The best Alexa Skills and commands: The ultimate Amazon Echo tips and tricks. TechRadar. Retrieved December 6, 2017 from https://www.techradar.com/how-to/the-best-amazon-echoalexa-skills-and-how-to-install-them.

[29] Christina N. Harrington, Kristin J. Hare, and Wendy A. Rogers. 2017. Developing a quick-start guide to aid older adults in interacting with gesture-based video games. Proceedings of the Human Factors and Ergonomics Society Annual Meeting 61, 1 (2017), 32-36. DOI : https://doi.org/10.1177/1541931213601503

[30] Julia Himmelsbach, Markus Garschall, Sebastian Egger, Susanne Steffek, and Manfred Tscheligi. 2015. Enabling accessibility through multimodality? interaction modality choices of older adults. In Proceedings of the 14th International Conference on Mobile and Ubiquitous Multimedia (MUM'15). 195-199. DOI : https://doi.org/10.1145/2836041.2836060

[31] Daniel J. Hruschka, Deborah Schwartz, Daphne Cobb St. John, Erin Picone-Decaro, Richard A. Jenkins, and James W. Carey. 2004. Reliability in coding open-ended data: Lessons learned from HIV behavioral research. Field Methods 16, 3 (2004), 307-331. DOI : https://doi.org/10.1177/1525822X04266540

[32] Lesa Huber and Carol Watson. 2014. Technology: Education and training needs of older adults. Educational Gerontology 40, 1 (2014), 16-25. DOI: https://doi.org/10.1080/03601277.2013.768064

[33] Matthew Jamieson, Brian O’Neill, Breda Cullen, Marilyn Lennon, Stephen Brewster, and Jonathan Evans. 2017. ForgetMeNot: Active reminder entry support for adults with acquired brain injury. In Proceedings of the $2017 \mathrm{CHI}$ Conference on Human Factors in Computing Systems. 6012-6023. DOI : https://doi.org/10.1145/3025453.3025888

[34] Jakob D. Jensen, Andy J. King, LaShara A. Davis, and Lisa M. Guntzviller. 2010. Utilization of internet technology by low-income adults: The role of health literacy, health numeracy, and computer assistance. fournal of Aging and Health 22, 6 (2010), 804-826. DOI : https://doi.org/10.1177/0898264310366161

[35] Heekyoung Jung, Heather Wiltse, Mikael Wiberg, and Erik Stolterman. 2017. Metaphors, materialities, and affordances: Hybrid morphologies in the design of interactive artifacts. Design Studies 53 (2017), 24-46. DOI: https: //doi.org/10.1016/J.DESTUD.2017.06.004

[36] Evangelos Karapanos, Jean-Bernard Martens, and Marc Hassenzahl. 2010. On the retrospective assessment of users' experiences over time. In Proceedings of the 28th of the International Conference Extended Abstracts on Human Factors in Computing Systems (CHI EA'10). 4075-4080. DOI : https://doi.org/10.1145/1753846.1754105

[37] Bran Knowles and Vicki L. Hanson. 2018. Older adults' deployment of 'distrust.' ACM Transactions on ComputerHuman Interaction 25, 4 (2018), Article 21. DOI : https://doi.org/10.1145/3196490

[38] Amanda Lazar, Mark Diaz, Robin Brewer, Chelsea Kim, and Anne Marie Piper. 2017. Going gray, failure to hire, and the ick factor: Analyzing how older bloggers talk about ageism. In Proceedings of the 2017 ACM Conference on Computer Supported Cooperative Work and Social Computing. 655-668. DOI : https://doi.org/10.1145/2998181.2998275

[39] Amanda Lazar, Christian Koehler, Joshua Tanenbaum, and David H. Nguyen. 2015. Why we use and abandon smart devices. In Proceedings of the 2015 ACM International foint Conference on Pervasive and Ubiquitous Computing (UbiComp'15). 635-646. DOI : https://doi.org/10.1145/2750858.2804288

[40] Chaiwoo Lee and Joseph F. Coughlin. 2015. PERSPECTIVE: Older adults' adoption of technology: An integrated approach to identifying determinants and barriers. Journal of Product Innovation Management 32, 5 (2015), 747-759. DOI : https://doi.org/10.1111/jpim.12176

[41] Q. Vera Liao and Wai-Tat Fu. 2014. Age differences in credibility judgments of online health information. ACM Transactions on Computer-Human Interaction 21, 1 (2014), Article 2. DOI : https://doi.org/10.1145/2534410 
[42] Siân E. Lindley, Richard Harper, and Abigail Sellen. 2009. Desiring to be in touch in a changing communications landscape: Attitudes of older adults. In Proceedings of the SIGCHI Conference on Human Factors in Computing Systems. 1693-1702. DOI : https://doi.org/10.1145/1518701.1518962

[43] Irene Lopatovska and Harriet Williams. 2018. Personification of the amazon alexa: BFF or a mindless companion? In Proceedings of the 2018 Conference on Human Information Interaction \& Retrieval. 265-268. DOI : https://doi.org/10. $1145 / 3176349.3176868$

[44] Taylor Martin. 2016. 50 Alexa skills worth using-CNET. Retrieved December 6, 2017 from https://www.cnet.com/ how-to/amazon-echo-most-useful-alexa-skills/.

[45] Roisin McNaney, John Vines, Daniel Roggen, Madeline Balaam, Pengfei Zhang, Ivan Poliakov, Patrick Olivier, Rísin McNaney, John Vines, Daniel Roggen, Madeline Balaam, Pengfei Zhang, Ivan Poliakov, and Patrick Olivier. 2014. Exploring the acceptability of google glass as an everyday assistive device for people with parkinson's. In Proceedings of the SIGCHI Conference on Human Factors in Computing Systems (CHI'14). 2551-2554. DOI : https://doi.org/10.1145/ 2556288.2557092

[46] Indrani Medhi, Somani Patnaik, Emma Brunskill, S.N. Nagasena Gautama, William Thies, and Kentaro Toyama. 2011. Designing mobile interfaces for novice and low-literacy users. ACM Transactions on Computer-Human Interaction 18, 1 (2011), Article 2. DOI : https://doi.org/10.1145/1959022.1959024

[47] Stephanie Medlock, Saeid Eslami, Marjan Askari, Derk L. Arts, Danielle Sent, Sophia E. De Rooij, and Ameen AbuHanna. 2015. Health information-seeking behavior of seniors who use the internet: A survey. Fournal of Medical Internet Research 17, 1 (2015), e10. DOI : https://doi.org/10.2196/jmir.3749

[48] Oussama Metatla, Alison Oldfield, Taimur Ahmed, Antonis Vafeas, and Sunny Miglani. 2019. Voice user interfaces in schools: Co-designing for Inclusion with visually-impaired and sighted pupils. In Proceedings of the 2019 CHI Conference on Human Factors in Computing Systems (CHI'19). Paper 378. DOI : https://doi.org/10.1145/3290605.3300608

[49] Momoko Nakatani, Takehiko Ohno, Ai Nakane, Akinori Komatsubara, and Shuji Hashimoto. 2012. How to motivate people to use internet at home: Understanding the psychology of non-active users. In Proceedings of the 2012 Asia Pacific Conference on Computer-Human Interaction (APCHI'12). 259-268. DOI : https://doi.org/10.1145/2350046.2350100

[50] Jakob Nielsen. 1994. 10 Usability Heuristics for User Interface Design. Retrieved May 14, 2019 from https://www. nngroup.com/articles/ten-usability-heuristics/.

[51] Tara Renee O’Brien, Frank Treiber, Carolyn Jenkins, and Angela Mercier. 2014. Use of computer and cellular phone technology by older rural adults. CIN: Computers Informatics Nursing 32, 8 (2014), 390-396. DOI : https://doi.org/10. 1097/CIN.0000000000000080

[52] Douglas A. Orr and Laura Sanchez. 2018. Alexa, did you get that? Determining the evidentiary value of data stored by the amazon ${ }^{\circledR}$ echo. Digital Investigation 24 (2018), 72-78. DOI : https://doi.org/10.1016/j.diin.2017.12.002

[53] Andrew Perrin and Jingjing Jiang. 2018. About a quarter of U.S. adults say they are 'almost constantly' online. Pew Research Center. Retrieved September 10, 2018 from http://www.pewresearch.org/fact-tank/2018/03/14/abouta-quarter-of-americans-report-going-online-almost-constantly/.

[54] Pew Research Center. 2018. Mobile fact sheet. Pew Research Center. Retrieved from http://www.pewinternet.org/factsheet/mobile/.

[55] Sam Pinney and James Laird. 2017. Best Alexa Skills: 17 Essential Skills for Your New Amazon Echo Speaker | Trusted Reviews. Retrieved December 6, 2017 from http://www.trustedreviews.com/news/alexa-skills-amazon-echo- 2946222

[56] Anne Marie Piper, Robin Brewer, and Raymundo Cornejo. 2017. Technology learning and use among older adults with late-life vision impairments. Universal Access in the Information Society 16, 3 (2017), 699-711. DOI : https://doi. org/10.1007/s10209-016-0500-1

[57] Anne Marie Piper, Nadir Weibel, and James D. Hollan. 2013. Audio-enhanced paper photos: Encouraging social interaction at age 105. In Proceedings of the 2013 Conference on Computer Supported Cooperative Work. 215-224. DOI : https://doi.org/10.1145/2441776.2441802

[58] Carlos Galinho Pires, Fernando Miguel Pinto, Vítor Duarte Teixeira, João Freitas, and Miguel Sales Dias. 2012. Living home center-A personal assistant with multimodal interaction for elderly and mobility impaired e-inclusion. Proceedings of the International Conference on Computational Processing of the Portuguese Language (PROPOR'12).

[59] Martin Porcheron, Joel E Fischer, Stuart Reeves, and Sarah Sharples. 2018. Voice interfaces in everyday life. In Proceedings of the 2018 CHI Conference on Human Factors in Computing Systems. Paper 640. DOI : https://doi.org/doi.org/ $10.1145 / 3173574.3174214$

[60] François Portet, Michel Vacher, Caroline Golanski, Camille Roux, and Brigitte Meillon. 2013. Design and evaluation of a smart home voice interface for the elderly: Acceptability and objection aspects. Personal and Ubiquitous Computing 17, 1 (2013), 127-144. DOI : https://doi.org/10.1007/s00779-011-0470-5

[61] Alisha Pradhan, Kanika Mehta, and Leah Findlater. 2018. "Accessibility came by accident": Use of voice-controlled intelligent personal assistants by people with disabilities. In Proceedings of the 2018 CHI Conference on Human Factors in Computing Systems. Paper 459. DOI : https://doi.org/10.1145/3173574.3174033 
[62] Amanda Purington, Jessie G. Taft, Shruti Sannon, Natalya N. Bazarova, and Samuel Hardman Taylor. 2017. "Alexa is my new BFF": Social roles, user satisfaction, and personification. In Proceedings of the 2017 CHI Conference Extended Abstracts on Human Factors in Computing Systems (CHI EA'17). 2853-2859. DOI: https://doi.org/10.1145/3027063. 3053246

[63] Agha Ali Raza, Bilal Saleem, Shan Randhawa, Zain Tariq, Awais Athar, Umar Saif, and Roni Rosenfeld. 2018. Baang: A viral speech-based social platform for under-connected populations. In Proceedings of the 2018 CHI Conference on Human Factors in Computing Systems (CHI'18). Paper 643. DOI : https://doi.org/10.1145/3173574.3174217

[64] Simon Robinson, Jennifer Pearson, Shashank Ahire, Rini Ahirwar, Bhakti Bhikne, Nimish Maravi, and Matt Jones. 2018. Revisiting "hole-in-the-wall" computing: private smart speakers and public slum settings. In Proceedings of the 2018 CHI Conference on Human Factors in Computing Systems (CHI'18). Paper 498. DOI : https://doi.org/10.1145/ 3173574.3174072

[65] Yvonne Rogers, Jeni Paay, Margot Brereton, Kate L. Vaisutis, Gary Marsden, and Frank Vetere. 2014. Never too old: Engaging retired people inventing the future with makey makey. In Proceedings of the SIGCHI Conference on Human Factors in Computing Systems. 3913-3922. DOI : https://doi.org/10.1145/2556288.2557184

[66] Frank Rudzicz, Rosalie Wang, Momotaz Begum, and Alex Mihailidis. 2015. Speech interaction with personal assistive robots supporting aging at home for individuals with alzheimer's disease. ACM Transactions on Accessible Computing 7, 2 (2015), Article 6. DOI : https://doi.org/10.1145/2744206

[67] Daisuke Sato, Masatomo Kobayashi, Hironobu Takagi, Chieko Asakawa, and Jiro Tanaka. 2011. How voice augmentation supports elderly web users. In Proceedings of the 13th International ACM SIGACCESS Conference on Computers and Accessibility. 155-162. DOI : https://doi.org/10.1145/2049536.2049565

[68] Sergio Sayago, Barbara Barbosa Neves, and Benjamin R. Cowan. 2019. Voice assistants and older people: some open issues. In Proceedings of the 1st International Conference on Conversational User Interfaces. Paper 7. DOI : https://doi. org/10.1145/3342775.3342803

[69] Laura Sbaffi and Jennifer Rowley. 2017. Trust and credibility in web-based health information: A review and agenda for future research. Journal of Medical Internet Research 19, 6 (2017), e218. DOI : https://doi.org/10.2196/jmir.7579

[70] S. Schlögl, G. Chollet, M. Garschall, M. Tscheligi, and G. Legouverneur. 2013. Exploring voice user interfaces for seniors. In Proceedings of the 6th International Conference on PErvasive Technologies Related to Assistive Environments (PETRA'13). Paper 52. DOI : https://doi.org/10.1145/2504335.2504391

[71] Julia Schwarz and Meredith Morris. 2011. Augmenting web pages and search results to support credibility assessment. In Proceedings of the 2011 Annual Conference on Human Factors in Computing Systems (CHI'11). 1245-1254. DOI : https: //doi.org/10.1145/1978942.1979127

[72] Joseph Sharit, Mario A. Hernández, Sara J. Czaja, and Peter Pirolli. 2008. Investigating the roles of knowledge and cognitive abilities in older adult information seeking on the web. ACM Transactions on Computer-Human Interaction 15, 1 (2008), Article 3. DOI : https://doi.org/10.1145/1352782.1352785

[73] Ben Shneiderman. The Eight Golden Rules of Interface Design. Retrieved May 14, 2019 from https://www.cs.umd. edu/ ben/goldenrules.html.

[74] Elizabeth Sillence, Pam Briggs, Lesley Fishwick, and Peter Harris. 2004. Trust and mistrust of online health sites. In Proceedings of the 2004 Conference on Human Factors in Computing Systems (CHI'04). 663-670. DOI : https://doi.org/10. 1145/985692.985776

[75] Amanda L. Smith and Barbara S. Chaparro. 2015. Smartphone text input method performance, usability, and preference with younger and older adults. Human Factors 57, 6 (2015), 1015-1028. DOI: https://doi.org/10.1177/ 0018720815575644

[76] Daniel Smolyak, Bongshin Lee, and Eun Kyoung Choe. 2018. TandemTrack. In Proceedings of the 2018 CHI Conference Extended Abstracts on Human Factors in Computing Systems. Paper LBW543. DOI : https://doi.org/10.1145/3170427. 3188536

[77] Yuling Sun, Silvia Lindtner, Xianghua Ding, Tun Lu, and Ning Gu. 2015. Reliving the past \& making a harmonious society today: A study of elderly electronic hackers in china. In Proceedings of the 18th ACM Conference on Computer Supported Cooperative Work \& Social Computing. 44-55. DOI : https://doi.org/10.1145/2675133.2675195

[78] Gokhan Tur, Asli Celikyilmaz, Xiaodong He, Dilek Hakkani-Tür, and Li Deng. 2018. Deep Learning in Conversational Language Understanding. Springer Singapore. DOI : https://doi.org/10.1007/978-981-10-5209-5_2

[79] Michel Vacher, Benjamin Lecouteux, Dan Istrate, Thierry Joubert, François Portet, Mohamed Sehili, and Pedro Chahuara. 2013. Experimental evaluation of speech recognition technologies for voice-based home automation control in a smart home. In Proceedings of the 4th Workshop on Speech and Language Processing for Assistive Technologies. 99-105.

[80] Eleftheria Vaportzis, Maria Giatsi Clausen, and Alan J. Gow. 2017. Older adults perceptions of technology and barriers to interacting with tablet computers: A focus group study. Frontiers in Psychology 8 (2017), Article 1687. DOI : https: //doi.org/10.3389/fpsyg.2017.01687 
[81] John Vines, Mark Blythe, Paul Dunphy, Vasilis Vlachokyriakos, Isaac Teece, Andrew Monk, and Patrick Olivier. 2012. Cheque mates: Participatory design of digital payments with eighty somethings. In Proceedings of the SIGCHI Conference on Human Factors in Computing Systems. 1189-1198. DOI : https://doi.org/10.1145/2208516.2208569

[82] John Vines, Gary Pritchard, Peter Wright, Patrick Olivier, and Katie Brittain. 2015. An age-old problem: Examining the discourses of ageing in HCI and strategies for future research. ACM Transactions on Computer-Human Interaction 22, 1 (2015), Article 2. DOI : https://doi.org/10.1145/2696867

[83] Alexandra Vtyurina and Adam Fourney. 2018. Exploring the role of conversational cues in guided task support with virtual assistants. In Proceedings of the 2018 CHI Conference on Human Factors in Computing Systems (CHI'18). Paper 208. DOI : https://doi.org/10.1145/3173574.3173782

[84] David Warnock, Marilyn McGee-Lennon, and Stephen Brewster. 2013. Multiple notification modalities and older users. In Proceedings of the SIGCHI Conference on Human Factors in Computing Systems. 1091-1094. DOI : https://doi. org/10.1145/2470654.2466139

[85] Zhuxiaona Wei and James A. Landay. 2018. Evaluating speech-based smart devices using new usability heuristics. IEEE Pervasive Computing 17, 2 (2018), 84-96. DOI : https://doi.org/10.1109/MPRV.2018.022511249

[86] Krzysztof Willman. 2017. 25 Best Amazon Echo Skills in the Alexa App | TurboFuture. Retrieved December 6, 2017 from https://turbofuture.com/consumer-electronics/The-20-Best-Amazon-Echo-Skills-in-the-Alexa-App.

[87] Maria K. Wolters, Christine Johnson, Pauline E. Campbell, Christine G. De Placido, and Brian McKinstry. 2014. Can older people remember medication reminders presented using synthetic speech? fournal of the American Medical Informatics Association 22, 1 (2014), 35-42. DOI : https://doi.org/10.1136/amiajnl-2014-002820

[88] Maria Klara Wolters, Fiona Kelly, and Jonathan Kilgour. 2016. Designing a spoken dialogue interface to an intelligent cognitive assistant for people with dementia. Health Informatics fournal 22, 4 (2016), 854-866. D0I : https://doi.org/ $10.1177 / 1460458215593329$

[89] Linda Wulf, Markus Garschall, Julia Himmelsbach, and Manfred Tscheligi. 2014. Hands free-care free: Elderly people taking advantage of speech-only interaction. In Proceedings of the 8th Nordic Conference on Human-Computer Interaction: Fun, Fast, Foundational. 203-206. DOI : https://doi.org/10.1145/2639189.2639251

[90] Eric Zeng, Shrirang Mare, and Franziska Roesner. 2017. End user security \& privacy concerns with smart homes. In Proceedings of the Symposium on Usable Privacy and Security (SOUPS'17).

Received February 2019; revised November 2019; accepted November 2019 